\title{
Modeling past plant species' distributions in mountainous areas: A way to improve our knowledge of future climate change impacts?
}

Louis François ${ }^{1}$ and Alain Hambuckers ${ }^{2}$

\begin{abstract}
Paleovegetation modeling, particularly in mountainous areas, could be an important method to understand and produce more reliable forecasts of the impacts of climate change on vegetation and plant species' distribution. The two main tools used for such studies are dynamic vegetation models and species-distribution models.
\end{abstract}

Greenhouse gas emissions and climate change are, without doubt, affecting plant species' distributions. The diversity of individual responses, the complexity of biotic interactions, and the intensity of human perturbations (fragmentation, pollution, hunting, etc.) make predictions for the future challenging. High-altitude mountain vegetation is particularly at risk and therefore interesting to study because, in the mountains, the climate gradients are steep and it is impossible for species growing near the summits to migrate to higher altitudes.

One of the keys to understand mountain species dynamics and assess future changes in their distributions, productivity and competitiveness, may be searched for in the past. Recent studies (e.g. Cheddadi et al. 2017; Pinaya et al. 2019) have shown that mountain tree species in Africa and South America have responded strongly to late Pleistocene and Holocene climate fluctuations. Here, we briefly examine whether current vegetation and plant-species models are appropriate tools to study those problems, and what the necessary requirements are to model mountain tree species' turnover observed in connection with paleoclimatic changes.

\section{The models and their data}

The most popular tools are the speciesdistribution models (SDMs; Fig. 1). They establish empirical relationships between species' presence and a set of predictors (climate variables, soil properties, land use, etc.; Thuiller et al. 2009). Vegetation can also be modeled with dynamic vegetation models (DVMs), which are process-based models describing the establishment, growth, and mortality of objects representing various plant types, competition among these objects, and processes such as photosynthesis, and respiration (Snell et al. 2014). Most often these objects are plant functional types corresponding to broad vegetation units, but it is also possible to simulate taxa, like species or genus. SDMs and DVMs require large amounts of climatic and environmental data with high enough resolution and accuracy. DVMs used with species require validation data sets like net primary productivity, which are more difficult to obtain.

In mountains, the accuracy of the forcing and validation data represents a challenge. Climate is strongly variable in space and time and it has been ascertained that growth and establishment of species are strongly dependent on local microclimatic and topographic features. The first challenge is thus to identify those conditions, by developing gridded climatic datasets at high spatial resolution over the studied areas. This is not an easy task, since not only temperature is affected by elevation and complex topography, but also precipitation and cloudiness, solar radiation, air humidity, and wind speed. All these variables must be reconstructed in a consistent way despite the scarcity of meteorological stations in high elevation

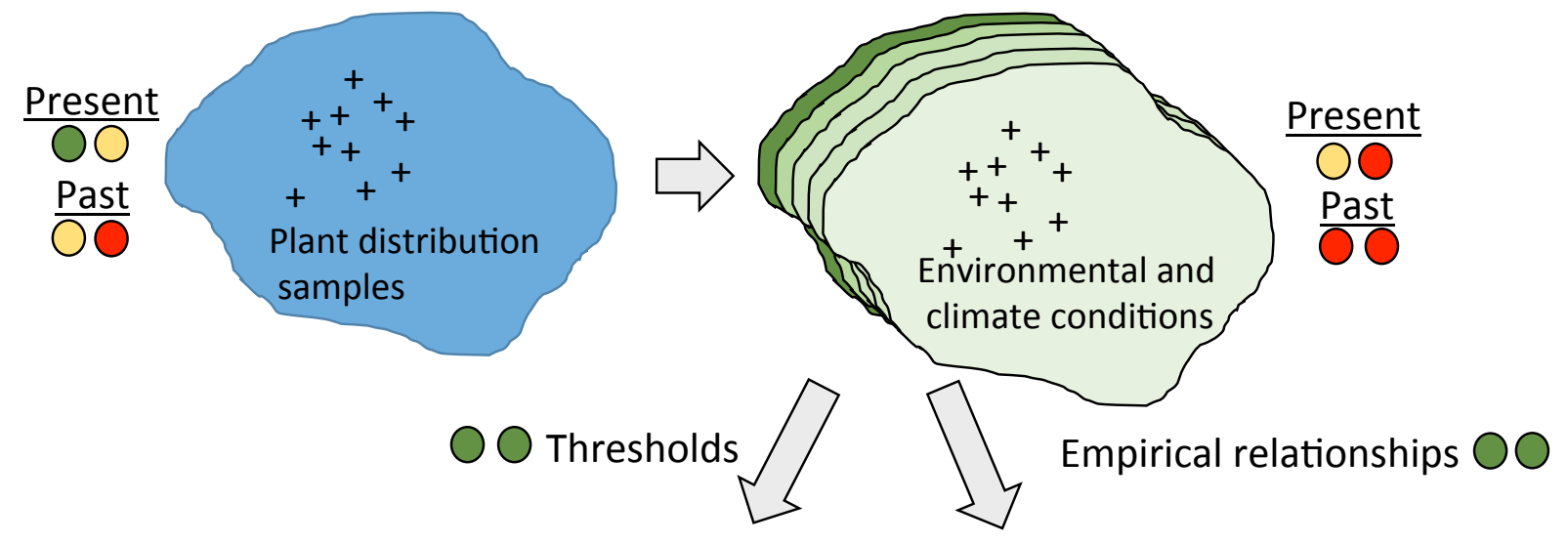

Processes quantification O Traits

\begin{tabular}{|l|}
\hline Possible improvements \\
\hline Migration $\bigcirc \bigcirc$ \\
Biotic interactions $\bigcirc \bigcirc$ \\
$\mathrm{CO}_{2}$ sensitivity $\bigcirc$ \\
Validation $\mathrm{O}$ \\
Trait variations $\bigcirc \bigcirc$
\end{tabular}

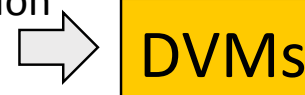

Outputs
Occupancy
Diversity
Vegetation
NPP, Biomass, etc.
Hydrology, etc.

\section{SDMs}

\begin{tabular}{|l|l|}
\hline Outputs & Possible improvements \\
\hline Occupancy & Migration $\mathrm{O}$ \\
Diversity & Biotic interactions $\mathrm{O}$ \\
Vegetation & $\mathrm{CO}_{2}$ sensitivity $\mathrm{O}$ \\
Validation $\mathrm{O}$
\end{tabular}




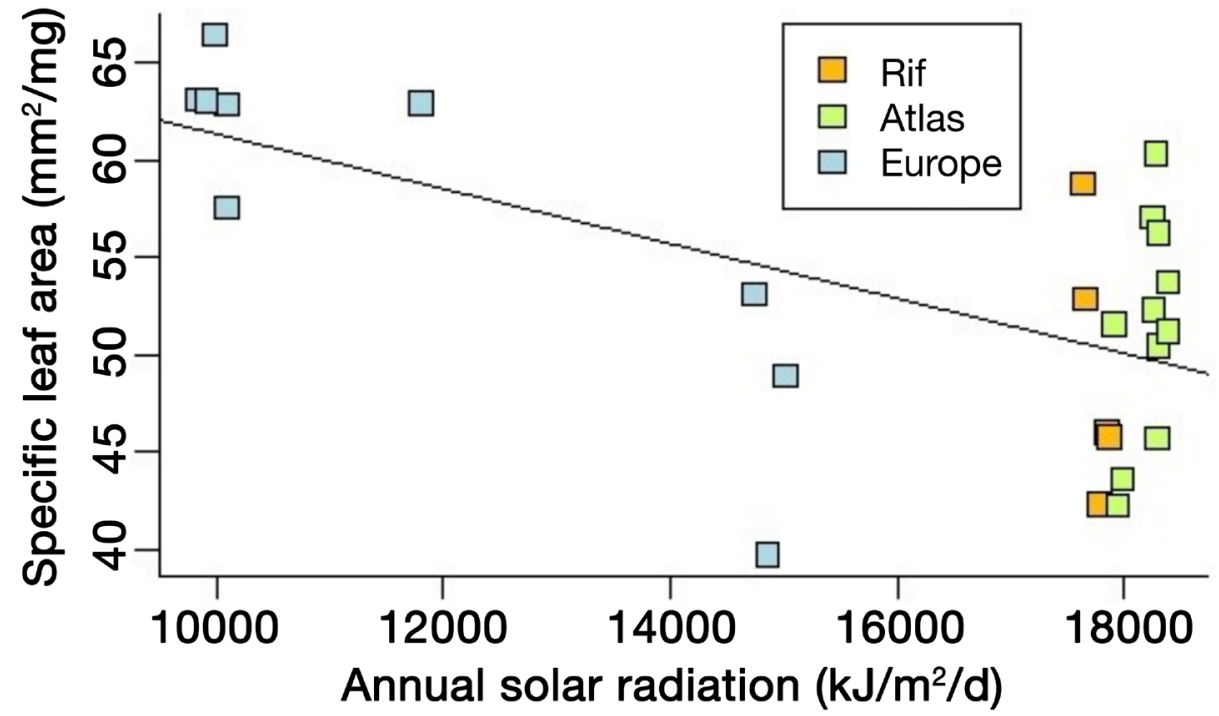

Figure 2: Example of trait variation with a climatic factor: specific leaf area of Cedrus atlantica in Morocco (Rif and Atlas Ranges) and in Europe as a function of annual solar radiation (linear mixed model; likelihood ratio test: $p$-value $=0.0006$, marginal $\left.R^{2}=0.5123\right)$. Other factors related to water availability also exert a control on this trait and may explain the large dispersion of the data points, making predictions challenging. Trait variation in time may follow similar behavior.

areas. Temperature inversions are also frequent, especially in winter, and the use of an average vertical temperature gradient may be problematic (Yang et al. 2011). Ideally, dynamic downscaling with high-resolution regional climate models should be used, but it is still rarely the case in ecological studies (Maclean 2019), especially where paleoclimatic reconstructions are concerned.

Complex topography also impacts soil hydrological conditions because of water transport along slopes. The water flow establishes hydrological gradients between mountain summits, and valleys, which induces changes in water-table depth. The presence of springs and the access to or capillary rise of groundwater may be critical for species survival during droughts; this has been found to be essential in explaining the distribution of vegetation at high spatial resolution (at scales smaller than a few kilometers) on any type of terrain. For that reason, SDMs and DVMs should take into account the water transport among grid cells and possibly the dynamics of the water table, when they target a very high spatial resolution. This approach, however, requires detailed and accurate information on soil depth, soil texture, and underlying rock porosity (Wolf 2011), generally not available over the entire distribution area of typical plant species.

\section{$\mathrm{CO}_{2}$ and plant traits}

The atmospheric $\mathrm{CO}_{2}$ level is another environmental variable that controls plant growth and distribution. The mixing ratio of $\mathrm{CO}_{2}$ in the atmosphere ( 410 ppmv in 2019) has shown very large fluctuations in the past: 1000 to 2000 ppmv in the Eocene, 190 ppmv during the Last Glacial Maximum, and 280 ppmv in the pre-industrial era. It has been established that elevated $\mathrm{CO}_{2}$ stimulates plant growth and resistance to hydric stress at least in the short term. Only DVMs are able to integrate these effects.
While DVMs seem more appropriate than SDMs to simulate past plant distribution, particularly in view of their sensitivity to $\mathrm{CO}_{2}$, their use is not straightforward, because this requires information on the morphophysiological properties of the taxa, the so-called plant traits. Examples of important traits are leaf and wood nitrogen contents, specific leaf area (i.e. area-to-weight ratio), and leaf longevity. Today, trait data are implemented in international databases, such as TRY (Kattge et al. 2020). However, they are still lacking for many species and the collection of such data is time consuming. In addition, many traits are sensitive to climate and envi ronmental conditions (Fig. 2), reflecting the acclimation capacity of the organisms.

Thus, there may have been variations in these traits in the past, modifying the species' sensitivity to climate variables. The involved processes are complex and consequently large uncertainties remain. For instance, this is the case with the long-term impacts of high $\mathrm{CO}_{2}$ levels (Van Der Sleen et al. 2015). $\mathrm{CO}_{2}$ enters the leaf through the stomata. This inflow increases under elevated $\mathrm{CO}_{2}$, which stimulates photosynthesis. But carbon fixation by photosynthesis also depends on leaf nitrogen, and it has been observed that, in the long term, the plant acclimates to elevated $\mathrm{CO}_{2}$ by allocating less nitrogen to leaves, which reduces the productivity enhancement. Also, stomatal density declines under elevated $\mathrm{CO}_{2}$, a phenomenon used to evaluate paleo- $\mathrm{CO}_{2}$ from fossil leaves (Royer 2001), which finally increases plant resistance to water stress and impacts the distribution.

\section{The biotic interactions}

Beyond the problems of physiology, additional complications in predicting the past or the future are associated with biotic interactions (competition-facilitation, predationparasitism) and migration limitations. The ranges of environmental conditions in which the species are able to thrive (fundamental niche) are modified by these biotic interactions. Species could also be absent in suitable conditions owing to dispersal limitations. SDMs include both, since they straightforwardly and statistically reflect the species-distribution sample used for their computation. This explains their ability to reproduce the present distribution, but also the challenge in predicting the past or the future. Indeed, SDMs rely on the hypothesis that biotic interactions and dispersal limitations are temporally constant. With species, DVMs reflect more the fundamental niche because the environmental thresholds limiting plant growth are derived from extreme supported values (Raghunathan et al. 2019). Obtaining the realized niche distributions requires long computation times with other plants to allow for competition for light and water to equilibrate.

Moreover, when making past or future projections, both modeling approaches produce new areas of climatic suitability but are rarely able to determine whether these areas could be reached by the organisms from their initial range. A first approach consists of using cellular automaton constraining cell occupancy of the new suitability areas with dispersal factors (Engler and Guisan 2009). The second approach is the coupling, with the plant models, of mechanistic models of seed dispersal by wind or animals. The main difficulty may be the discrepancy between the scales of the processes, since the dispersal model works for individual plants, while the resolution of plant models is typically on the order of at least several kilometers. Both methods could be used with DVMs or SDMs but data are lacking particularly for validation.

In conclusion, for achieving reliable reconstructions of paleovegetation, current models could be improved. However, it is mandatory to develop spatio-temporal series of data to allow for robust validation of these models.

\section{AFFILIATIONS}

'Unit for Modeling of Climate and Biogeochemical Cycles, UR SPHERES, University of Liège, Belgium ${ }^{2}$ Behavioural Biology Unit, UR SPHERES, University of Liège, Belgium

\section{CONTACT}

Louis François: louis.francois@uliege.be

\section{REFERENCES}

Cheddadi R et al. (2017) Front Ecol Environ 5: 114 Engler R, Guisan A (2009) Divers Distrib 15: 590-601 Kattge J et al. (2020) Glob Change Biol 26: 119-188 Maclean IMD (2019) Glob Change Biol 26: 1003-1011

Pinaya JLD et al. (2019) Sci Rep 9: 17912

Raghunathan N et al. (2019) Reg Environ Change 19: 219-232

Royer DL (2001) Rev Palaeobot Palynol 114: 1-28 Snell RS et al. (2014) Ecography 37: 1184-1197 Thuiller W et al. (2009) Ecography 32: 369-373 Van Der Sleen P et al. (2015) Nat Geosci 8: 24-28 Wolf A (2011) Ecol Modell 222: 2595-2605

Yang Z et al. (2011) Geogr Ann Ser A 93: 89-112 